\title{
Dipole-magnet field models based on a conformal map
}

\author{
P. L. Walstrom \\ Los Alamos National Laboratory, Los Alamos, New Mexico 87545, USA
}

(Received 24 August 2011; published 11 October 2012)

\begin{abstract}
In general, generation of charged-particle transfer maps for conventional iron-pole-piece dipole magnets to third and higher order requires a model for the midplane field profile and its transverse derivatives (soft-edge model) to high order and numerical integration of map coefficients. An exact treatment of the problem for a particular magnet requires use of measured magnetic data. However, in initial design of beam transport systems, users of charged-particle optics codes generally rely on magnet models built into the codes. Indeed, if maps to third order are adequate for the problem, an approximate analytic field model together with numerical map coefficient integration can capture the important features of the transfer map. The model described in this paper is based on the fact that, except at very large distances from the magnet, the magnetic field for parallel pole-face magnets with constant pole gap height and wide pole faces is basically two dimensional (2D). The field for all space outside of the pole pieces is given by a single (complex) analytic expression and includes a parameter that controls the rate of falloff of the fringe field. Since the field function is analytic in the complex plane outside of the pole pieces, it satisfies two basic requirements of a field model for higher-order map codes: it is infinitely differentiable at the midplane and also a solution of the Laplace equation. It is apparently the only simple model available that combines an exponential approach to the central field with an inverse cubic falloff of field at large distances from the magnet in a single expression. The model is not intended for detailed fitting of magnetic field data, but for use in numerical map-generating codes for studying the effect of extended fringe fields on higher-order transfer maps. It is based on conformally mapping the area between the pole pieces to the upper half plane, and placing current filaments on the pole faces. An algorithm for computing the midplane field derivatives with the model is described. The model has been incorporated in the particle beam code MARYLIE/IMPACT as a special dipole-magnet type along with a tanh model with exponential falloff of the fringe field. Comparison of maps from the tanh model and the new model shows that significant differences in 3rd-order geometric terms can occur, apparently due to the extended fringe field in the new model.
\end{abstract}

DOI: 10.1103/PhysRevSTAB.15.102401

PACS numbers: 41.20.Gz, 41.85.-p

\section{INTRODUCTION}

The field above and below the midplane of a 2D dipole can be reconstructed to arbitrary order if the midplane field and its longitudinal derivatives to the same order can be computed for arbitrary points on the midplane. These derivatives, together with the midplane vector potential, are the basic inputs to numerical map integration codes. Convergence of field expansions in the beam volume with the new model is guaranteed since effective sources are on the pole pieces (except for a small region outside of the pole gap-see Sec. VI). Among the computer codes with numerical map generation capability for bending dipoles with finite fringe-fields (soft-edge models) are MARYLIE [1], MARYLIE/IMPACT [2], and COSY INFINITY [3]. The softedge dipole models in MARYLIE and MARYLIE/IMPACT (MLI) are not presently well documented. Soft-edge

Published by the American Physical Society under the terms of the Creative Commons Attribution 3.0 License. Further distribution of this work must maintain attribution to the author(s) and the published article's title, journal citation, and DOI. rare-earth permanent quadrupole magnets and solenoids are documented in Ref. [1]. Numerical generation of maps for soft-edged dipoles in MARYLIE and associated codes are described in Ref. [4]; the new soft-edge MLI elements are documented in internal Los Alamos National Laboratory memoranda.

Numerical map integrators generally require the vector potential and the magnetic field and its longitudinal derivatives on the magnet midplane. In cases where the magnetic field is basically 2D, i.e., depends only on a longitudinal coordinate $x$ and an off-midplane coordinate $y$, the field in the space outside of coils and iron pole pieces can be described by an analytic function in the complex plane, i.e., one can write

$$
B_{y}+i B_{x}=b(x+i y)
$$

where $b(x)=B_{y}(x, 0)$. The off-axis field expansion in $y$, the distance from the midplane, can then be derived by Taylor expansion of $b(x+i y)$ in the variable $i y$ around $y=0$, with the result 


$$
\begin{gathered}
B_{x}(x, y)=y b^{\prime}(x)-\frac{y^{3}}{6} b^{\prime \prime \prime}(x)+\cdots(-1)^{n-1} \\
\times \frac{y^{2 n-1}}{(2 n-1) !} b^{[2 n-1]}(x) \cdots, \\
B_{y}(x, y)=b(x)-\frac{y^{2}}{2} b^{\prime \prime}(x)+\frac{y^{4}}{24} b^{\prime \prime \prime \prime}(x)-\cdots(-1)^{n-1} \\
\times \frac{y^{2 n-2}}{(2 n-2) !} b^{[2 n-2]}(x) \cdots
\end{gathered}
$$

Since higher-order transfer-map codes generally use canonical variables, an expression for vector potential is also needed. The natural vector potential for the present problem has a single component $A_{z}$, which is just the negative of the indefinite integral of $B_{y}$ with respect to $x$ at constant $y$ :

$$
A_{z}(x, y)=-\int_{-\infty}^{x} B_{y}\left(x^{\prime}, y\right) d x^{\prime} .
$$

This vector potential has the drawback that, even for a magnetic field distribution bounded in $x$, it is nonzero at large positive $x$. An alternative vector potential is $A_{x}(x, y, z)=z B_{y}(x, y), A_{y}(x, y, z)=-z B_{x}(x, y)$, and $A_{z}=$ 0 . Although the field of $2 \mathrm{D}$ dipoles does not vary in $z$, it is necessary to put a $z$ dependence into the vector potential in this gauge. Since the magnetic field for finite-length dipoles falls off to zero outside of the magnet, the kinetic and canonical momenta in this gauge approach the same limit at large distances from the magnet. On the other hand, although $A_{z}$ of Eq. (4) is finite after a bend, it is useful in determining the bending angle along the reference trajectory, and is sometimes required by higher-order map codes in modeling dipoles.

Besides the conformal-map dipole model of this paper, the only analytic field model available in MLI for 2D dipoles is a double tanh model. In the tanh model, the midplane field profile is taken to be

$$
b(x)=\frac{B_{0}}{2}\left[\tanh \frac{\pi}{2 a}(x+2 b)-\tanh \frac{\pi}{2 a} x\right] .
$$

In Eq. (5), the nominal pole width is $2 b$, the full pole gap $2 a$, and the right-hand pole face is at $x=0$. The central field approaches $B_{0}$ as $b / a$ becomes large. When extended to the complex plane, the tanh profile function is found to have singularities (simple poles) equally spaced along vertical lines at $x=-2 b$ and $x=0$. The poles closest to the midplane are at $y= \pm a$. The complex potential function that gives the tanh field function is $\log \cosh$, which corresponds to an array of current filaments of the same sign with equal spacing. At very large $|x|$, the arrays at $x=-2 b$ and $x=0$ form effective infinite current sheets of opposite sign and their contributions sum to zero. At intermediate distances, the field from either one of the arrays approaches the constant current-sheet field exponentially in distance from the array. The falloff of the combined field from the two source arrays in the fringefield regions therefore approaches zero exponentially in distance from either array.

The tanh profile is unrealistic in that the field profile has an exponential falloff in the fringe field at large $|x|$. In general, the fringe-field profile at the ends of wide dipole magnets is asymmetrical, with the field between the poles approaching the central field exponentially in distance from the pole edge, but falling off more slowly (typically with an inverse power of distance) for points outside of the gap. Another commonly used analytic model for fringefield profiles is Enge profile $E(x)=B_{0} /\left[1+e^{S(x)}\right]$, with $S(x)=c_{0}+c_{1} x+c_{2} x^{2}+c_{3} x^{3}$ [5]. With translation and reflection, the Enge profile can represent a double-sided field profile, i.e., $b(x)=E(x)+E(-x-2 b)-B_{0}$. If only the linear term is nonzero in the Enge expression, the field profile becomes the tanh profile with Enge's $c_{1}$ equal to $\pi / a$ in Eq. (5). When higher-order terms are added to $S(x)$, the fringe-field profile is asymmetric, but field falloff for large $x$ is faster than exponential: $b(x) \approx e^{-a_{N} x^{N}}$, where $a_{N}$ is the coefficient of the highest power of $x$ in $S(x)$. Note that $a_{N}$ must be positive. In the following, asymmetric 2D analytic field profiles are derived by use of conformal mapping of pole pieces and placement of current filaments on the pole pieces. These profiles have fringe fields with a negative power-law dependence on $|x|$ at large $|x|$, but with an exponential approach of the field to the central value inside of the pole faces.

\section{CONFORMAL MAP FOR SINGLE-SIDED POLE PIECES}

In this and the following sections, $z$ is the complex variable $x+i y$, not the coordinate perpendicular to the $x-y$ plane. The conformal map from the upper half of the $t$ plane, with $t=r+i s$ [see Fig. 1(b)], to the area in the $z$ plane outside of the poles of Fig. 1(a) is derived by use of the Schwartz-Christoffel transformation and can be found in many textbooks:

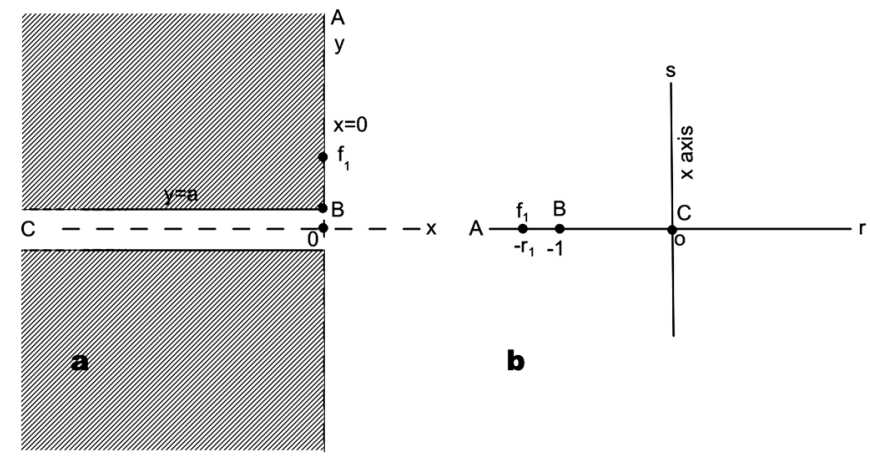

FIG. 1. Conformal mapping of one side of a semi-infinite dipole magnet to the upper half of the $t$ plane. (a) Pole pieces in the $z$ plane. (b) Image of pole pieces and $x$ axis in $t$ plane. $f_{1}$ indicates the position of a current filament on the pole pieces. 


$$
z=\frac{2 a}{\pi}\left[\sqrt{1-t^{2}}-\tanh ^{-1} \sqrt{1-t^{2}}\right]-i a .
$$

Equation (6) can be rewritten in the equivalent form

$$
z=\frac{2 a}{\pi}\left\{\sqrt{1-t^{2}}+\ln t-\ln \left[1+\sqrt{1-t^{2}}\right]\right\}-i a .
$$

As a check, inserting $t=-1$ into Eq. (7) gives $1-t^{2}=0$, $\ln t=i \pi$, and $z=a i$, as desired. Positive real values of $t$ map to the lower pole-piece surface. Negative real values of $t$ with small $|t|$ map to $2 a / \pi \ln |t|+i a$ and approach $-\infty+i a$ as $|t|$ approaches zero. Negative real values of $t$ with $t=-R, R>1$ map to the upper pole face $(x=0$, $y>a)$, with

$$
y=\frac{2 a}{\pi}\left[\sqrt{R^{2}-1}-\tan ^{-1} \sqrt{R^{2}-1}\right]+a .
$$

On the other hand, purely imaginary values of $t$ with $t=$ $i s, s>0$ and real, map to real values of $z$, with $y=0$ and

$$
x=\frac{2 a}{\pi}\left\{\sqrt{1+s^{2}}+\ln s-\ln \left[1+\sqrt{1+s^{2}}\right]\right\} .
$$

For small $s$, Eq. (9) gives $x \approx 2 a / \pi[\ln s+1-\ln 2]$, while for large $s$ it gives $x \approx 2 a s / \pi$. That is, the conformal map of Eq. (6) maps the upper imaginary half axis of the $t$ plane to the entire $x$ axis of the $z$ plane. Equation (8) will be used to find the value of $t$ for filament positions specified in the $z$ plane, while Eq. (9) will be used to find $t$ values corresponding to arbitrary $x$ values on the real axis during evaluation of $b(x)$ and its $x$ derivatives [see Eqs. (1)-(3)].

\section{CONFORMAL MAP FOR DOUBLE-SIDED POLE PIECES}

Conformal mapping from the upper half plane ( $t$ plane) to the area outside of two finite-width pole pieces in the $z$ plane (see Fig. 2) using the Schwartz-Christoffel transform was first worked out by Davy in 1944 [6] and involves elliptic integrals and elliptic functions. Davy's mapping is used in Ref. [7] to compute the integral of the square of the electric field along the midline between two rectangular

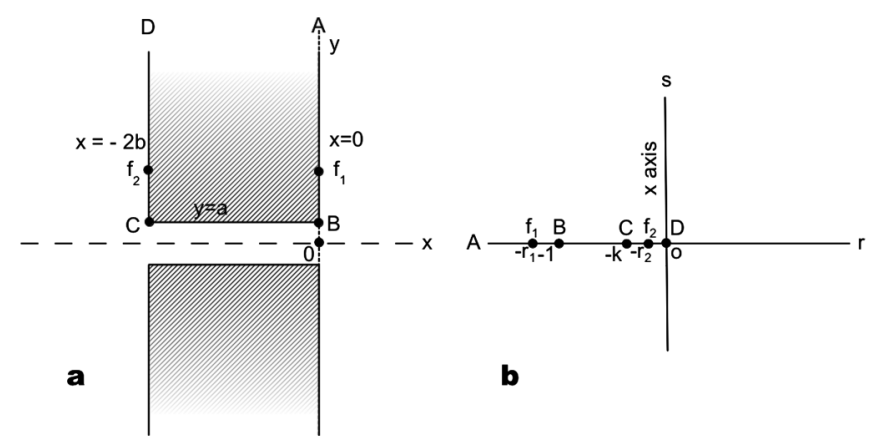

FIG. 2. Conformal mapping of double-sided pole pieces. (a) Pole pieces in the $z$ plane. (b) Image of pole pieces and $x$ axis in $t$ plane. $f_{1}$ and $f_{2}$ indicate the positions of current filaments on the pole pieces. electrodes. Real values of $t$ map to the pole-piece surfaces in the $z$ plane. Davy's conformal map is expressed as a function of the intermediate complex variable $u$, defined by $\operatorname{sn}(u, k)=1 / t$ :

$$
\begin{aligned}
x+i y= & \frac{a i}{2 E-k^{\prime 2} K}\left[\left(k^{\prime 2}-\frac{2 E}{K}\right) u-2 Z(u)\right. \\
& \left.-\frac{\operatorname{cn}(u) \operatorname{dn}(u)}{\operatorname{sn}(u)}\right]+T .
\end{aligned}
$$

The modulus of the Jacobian elliptic functions cn, dn, and sn, Jacobi's function $Z$, and of the complete elliptic integrals $E$ and $K$ in Eq. (10) is denoted here by $k$. The complete elliptic integrals $E, E^{\prime}, K$, and $K^{\prime}$ have real values that are a function $k$ only and are fixed for fixed $b / a$. The complementary modulus $k^{\prime}=\sqrt{1-k^{2}}, K^{\prime}(k)=K\left(k^{\prime}\right)$, and $E^{\prime}(k)=E\left(k^{\prime}\right)$. The modulus $k$ is the solution of the transcendental equation

$$
\frac{K^{\prime} k^{\prime 2}-2 K^{\prime}+2 E^{\prime}}{2\left(K k^{\prime 2}-2 E\right)}=\frac{b}{a}
$$

The modulus $k$ lies in the interval $[0,1]$ but is small for $b / a>1$. For $b / a=1, k \approx 0.023$; for $b / a=2, k \approx$ 0.001. To a good approximation, when $b / a>1, k \approx$ $4 e^{-2-\pi b / a}$. Equation (10) can be simplified somewhat by using the definition $Z(u)=E(u)-(E / K) u$ :

$$
x+i y=\frac{a i}{2 E-k^{\prime 2} K}\left[k^{\prime 2} u-2 E(u)-\frac{\operatorname{cn}(u) \operatorname{dn}(u)}{\operatorname{sn}(u)}\right]+T .
$$

In this section, $E$ without an argument is understood to be the complete elliptic integral of the second kind; when it appears with an argument as in Eq. (12), it is an incomplete elliptic integral and in general assumes complex values; i.e., $E(u)=E(u, k)=E(\theta+i \phi, k)$, where $E(\theta+i \phi, k)$ is the Legendre form of $E$. The angles $\theta$ and $\phi$ are in turn determined by the relation $F(\theta+i \phi, k)=u$. In this paper the real constant $T$ in Eqs. (10) and (12) is set to zero. Then, the right-hand corner of the upper pole piece is mapped to $z=i a$ and the left-hand corner to $z=-2 b+a i$. This choice of $z$-plane coordinate origin is convenient for direct comparison with the map of the single-sided pole pieces of the previous section.

As in the single-sided pole problem, the positive half of the imaginary axis in the $t$ plane is mapped to the entire $x$ axis in the $z$ plane. Writing sn $u=1 /(i s), s>0$ and real, we have am $u=-i \sinh ^{-1}(1 / s)$ and $u=$ $-F\left[i \sinh ^{-1}(1 / s), k\right]$. Using the expression for $F$ of imaginary angle (Equation 17.4.8 of Ref. [8]), we have $u=-i F\left(\tan ^{-1} 1 / s, k^{\prime}\right)$. Setting $\beta=\tan ^{-1}(1 / s), \quad u=$ $-i F\left(\beta, k^{\prime}\right)$ and $E(u)=-i E\left(\beta, k^{\prime}\right)+i F\left(\beta, k^{\prime}\right)+(i / s) \times$ $\left[\left(k^{2}+s^{2}\right) /\left(1+s^{2}\right)\right]^{1 / 2}$. Also, cn $u=\left(1+1 / s^{2}\right)^{1 / 2}$ and dn $u=\left(1+k^{2} / s^{2}\right)^{1 / 2}$. Putting all of this into Eq. (12), for $t=i$ s we get 


$$
\begin{aligned}
x= & \frac{a}{2 E-k^{\prime 2} K}\left[2 E\left(\beta, k^{\prime}\right)-\left(1+k^{2}\right) F\left(\beta, k^{\prime}\right)\right. \\
& \left.-\frac{2}{s}\left(\frac{k^{2}+s^{2}}{1+s^{2}}\right)^{1 / 2}+\frac{1}{s}\left(1+s^{2}\right)^{1 / 2}\left(k^{2}+s^{2}\right)^{1 / 2}\right] .
\end{aligned}
$$

For small $k$, the complete elliptical integrals $E$ and $K$ both approach $\pi / 2$ and $a /\left(2 E-k^{12} K\right) \approx 2 a / \pi$. For $s>k$ and $k$ small, $E\left(\beta, k^{\prime}\right) \approx 1 /\left(1+s^{2}\right)^{1 / 2}, F\left(\beta, k^{\prime}\right) \approx-\ln s+$ $\ln \left[1+\left(1+s^{2}\right)^{1 / 2}\right]$, and Eqs. (9) and (13) become identical. Numerical checks using Bullirsch algorithms [9] for the elliptic integrals verify this result and show negligible difference between the single-sided and double-sided mapping of the $x$ axis for $b / a>2$. Therefore, in the remainder of this paper, only the computationally simpler singlesided map of Eq. (7) together with reflection and translation of the single-sided field profiles derived in the following section will be used for computation of fields and field derivatives for double-sided magnets.

\section{CURRENT FILAMENT MODEL FOR THE FIELD}

If a filament carrying an electrical current $I$ is placed between the pole pieces at $x=-\infty$ in the $z$ plane of Fig. 1 , in the infinite permeability limit it will induce a difference in magnetic scalar potential between the lower and upper pole pieces of $\mu_{0} I$ (MKS units). In the $t$ plane, this filament has $t=0$. In the $t$ plane, the complex scalar potential for the single filament has the same form as that for a current filament in free space, except that the field is twice as large for the same current since the field lines traverse semicircles instead of full circles: $\Phi=\mu_{0} I / \pi \ln t$. The magnetic field in the $z$ plane is obtained by the chain rule of differentiation:

$$
B_{y}+i B_{x}=\frac{d \Phi}{d z}=\frac{d \Phi}{d t} \frac{d t}{d z} .
$$

For points on the $x$ axis, $t=i s$ and $B_{x}=0$. We then have $d \Phi / d t=\mu_{0} I /(i \pi s), d t / d z=(i \pi / 2 a)\left[s /\left(1+s^{2}\right)^{1 / 2}\right]$, and

$$
B_{y}=\frac{\mu_{0} I}{2 a} \frac{1}{\sqrt{1+s^{2}}} .
$$

Equation (15) is the usual expression for the fringe field of a rectangular-pole dipole magnet, or, except for the constant factor, the electric field between two semi-infinite rectangular electrodes. For points well inside the pole gap where $s$ is small, $B \approx \mu_{0} I /(2 a)$. For large positive $x, x \approx 2 a s / \pi$, and $B \approx \mu_{0} I /(\pi x)$. This field falloff is slower than that for real magnets and is a consequence of the fact that the pole pieces form equipotential surfaces that extend infinitely above and below the midplane.

In real dipole electromagnets, the end windings cross the pole faces parallel to the gap at some distance above and below the gap. Above the upper windings and below the lower windings, the magnetic scalar potential drops to zero. The essential features of this geometry can be modeled by placing current filaments on the faces of the upper and lower pole pieces (one such filament is shown in Fig. 1) at a distance $d$ from the pole corners. Each filament has a current of $-I / 2$. In the $z$ plane, the positions of the two filaments are $\pm i(a+d)$, and in the $t$ plane, the real values $\mp r_{1}$. Given $d, r_{1}$ is found by solving Eq. (8) for $R$ with $y=a+d$ and then setting $r_{1}=R$. In the $t$ plane, the complex scalar potential with the two added filaments is

$$
\Phi=\frac{\mu_{0} I}{\pi}\left[\ln t-\frac{1}{2} \ln \left(t+r_{1}\right)-\frac{1}{2} \ln \left(t-r_{1}\right)\right] .
$$

The single-sided midplane field profile is then

$$
f_{\mathrm{s}}(x)=\frac{\mu_{0} I}{2 a} \frac{r_{1}^{2}}{\left[r_{1}^{2}+s^{2}(x)\right] \sqrt{1+s^{2}(x)}} .
$$

For large positive $x$, the field falloff is now

$$
f_{\mathrm{s}}(x) \approx \frac{\mu_{0} I r_{1}^{2}}{2 a}\left(\frac{2 a}{\pi x}\right)^{3} .
$$

It is interesting that the inverse cubic dependence of the fringe field described by Eq. (18) has been achieved with a two-dimensional field model. More elaborate models can be constructed by placing additional filaments on the pole pieces, the only requirement being that the currents of all of the filaments on the pole pieces add up to $-I$.

As is usual in all but the most elementary conformal mapping problems, the mapping function is explicitly defined as $z(t)$, and closed-form expressions for $t(z)$ are not available. Values of $t$ for arbitrary $z$ must be obtained by numerical inversion of the mapping function. If only midplane field values and filament positions on the pole pieces are needed, solutions of transcendental equations in complex variables is not required for the inversion of the mapping function: Eqs. (9) and (8) involve only real quantities. In practice, Eqs. (9) and (8) are solved using Newton's method with starting values estimated by use of various simple approximate expressions for $t(z)$ for various regions of $z$.

As with the Enge profile, two single-sided fringe-field profiles can be combined by translation and reflection to construct a symmetrical field profile $b(x)$ suitable for modeling the field of finite-width dipoles:

$$
b(x)=f_{\mathrm{s}}(x)+f_{\mathrm{s}}(-x-2 b)-B_{0} .
$$

The profile of Eq. (19) models a dipole with the left-hand pole face at $x=-2 b$ and the right-hand pole face at $x=0$ and is useful for dipoles with length-to-gap ratio $b / a>2$, which covers most cases of interest. An analytic expression for the "natural" vector potential (negative of the indefinite integral of the midplane field) of the finite-width dipole is also found by combining two single-sided potentials by reflection and translation. Setting $t=i$ is in Eq. (16), one gets for the single-sided complex potential

$$
\Phi=\frac{\mu_{0} I}{\pi} \ln \frac{s}{\sqrt{s^{2}+r_{1}^{2}}} .
$$


On the midplane, the scalar potential is zero, and the complex potential has only a real component, which is the negative of the vector potential. Writing $\Phi(x)=$ $\Phi[s(x)]$, for large negative $x, \Phi(x)$ has the asymptotic form $\Phi(x)=B_{0} x+\mu_{0} I / \pi\left[\ln 2-1-\ln r_{1}\right]$, where $B_{0}=$ $\mu_{0} I / 2 a$. Then the indefinite integral of the midplane field for the double-sided magnet is

$$
\begin{aligned}
\int_{-\infty}^{x} B\left(x^{\prime}, 0\right) d x^{\prime}= & \Phi(x)-\Phi(-x-2 b)-B_{0} x \\
& -\frac{\mu_{0} I}{\pi}\left(\ln 2-1-\ln r_{1}\right) .
\end{aligned}
$$

The field integral for the entire magnet, which is the asymptotic value of $-A_{z}(x)$ for large positive $x$, is

$$
\int_{-\infty}^{+\infty} B(x, 0) d x=2 b B_{0}-2 \frac{\mu_{0} I}{\pi}\left(\ln 2-1-\ln r_{1}\right) .
$$

Since the second term in Eq. (22) is positive, the field integral is somewhat larger than $2 b B_{0}$.

\section{ALGORITHMS FOR COMPUTING HIGHER DERIVATIVES OF $\boldsymbol{b}(\boldsymbol{x})$}

If we set $f(s)$ equal to the right-hand side of Eq. (17), $g(s)=d s / d x=(\pi / 2 a)\left[s /\left(1+s^{2}\right)^{1 / 2}\right]$, and denote derivatives by primes, we can write

$$
\begin{array}{r}
f_{\mathrm{s}}(x)=f \\
f_{\mathrm{s}}^{\prime}(x)=g f^{\prime} \\
f_{\mathrm{s}}^{\prime \prime \prime}(x)=g\left(g f^{\prime}\right)^{\prime} \\
f_{\mathrm{s}}^{\prime \prime \prime}(x)=g\left(g\left(g f^{\prime}\right)^{\prime}\right)^{\prime} \\
\ldots \ldots \cdots \cdots \cdots \cdots \\
f_{\mathrm{s}}^{[n]}(x)=g\left(g\left(\cdots\left(g f^{\prime}\right)^{\prime} \cdots\right)^{\prime}\right)^{\prime} .
\end{array}
$$

The right-hand sides of Eq. (23) are the result of repeated application of the operator $g(s) d / d s$ to the right-hand side of the previous step and are functions of $s$ only. Explicit

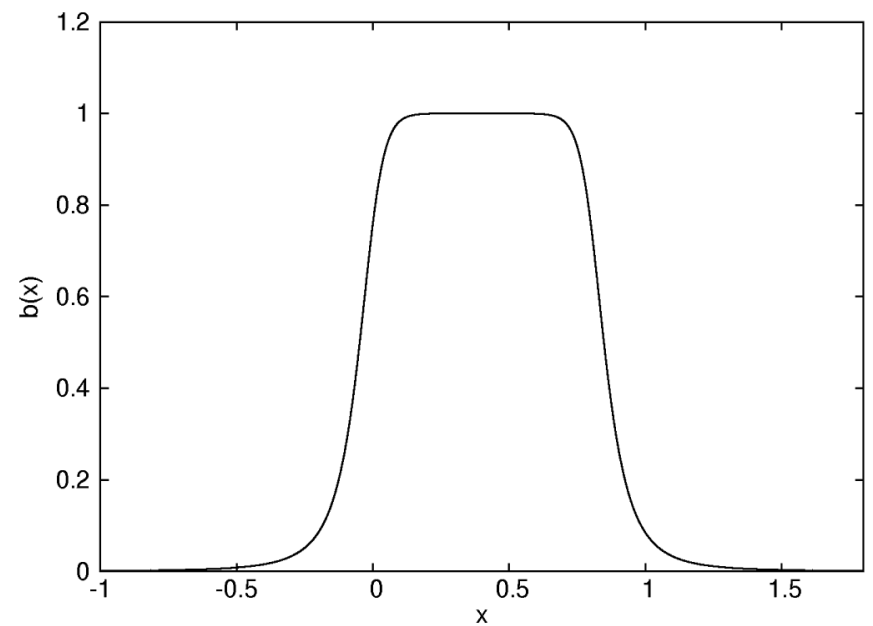

FIG. 3. Field profile for a dipole magnet with $a=0.1, b=$ $0.4, d / a=0.5$, and $B_{0}=1$.

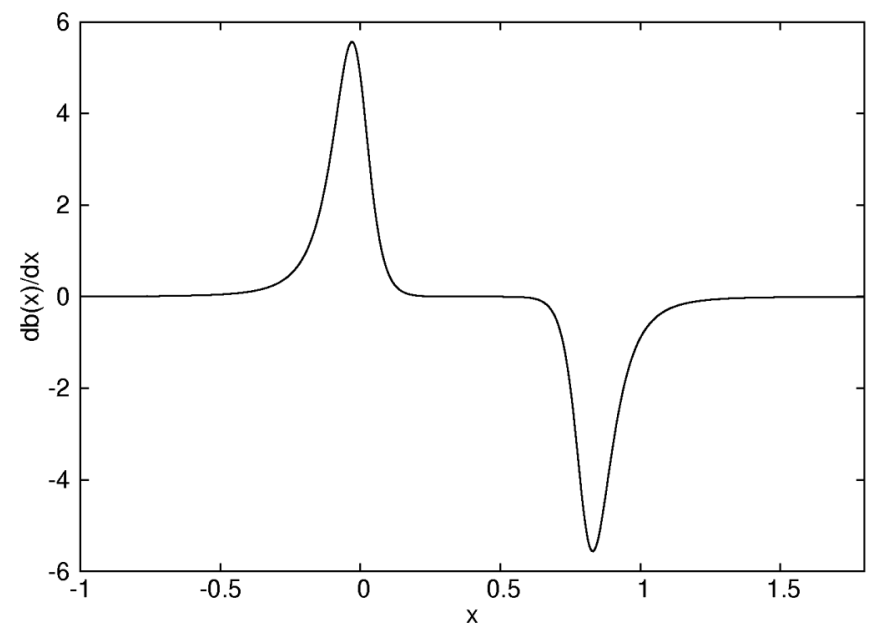

FIG. 4. First derivative of the field profile for a dipole magnet with $a=0.1, b=0.4, d / a=0.5$, and $B_{0}=1$.

expressions for the right-hand side to arbitrary order for arbitrary $f$ and $g$ can be obtained by use of a symbolic mathematics program such as MAXIMA to apply the operator multiple times and then expand the resultant expressions. After $s$ is found by numerical solution of Eq. (9), a computer subroutine to evaluate these expressions, together with subroutines to compute the $s$ derivatives of $f(s)$ and $g(s)$, gives the desired $x$ derivatives of $f_{\mathrm{s}}(x)$. Then the $n$th derivative of the field profile is given by

$$
b^{[n]}(x)=f_{\mathrm{s}}^{[n]}(x)+(-1)^{n} f_{\mathrm{s}}^{[n]}(-x-2 b) .
$$

A set of computer subroutines was written to evaluate $b(x)$ and its derivatives to 6th order using the above model. Instead of specifying the current $I$, the limiting field $B_{0}=\mu_{0} I / 2 a$ is specified. Figure 3 is a plot of $b(x)$ for a dipole with $a=0.1, b=0.4, d / a=0.5$, and $B_{0}=1$. Figures 4 and 5 are plots of $b^{\prime}(x)$ and $b^{\prime \prime}(x)$, respectively,

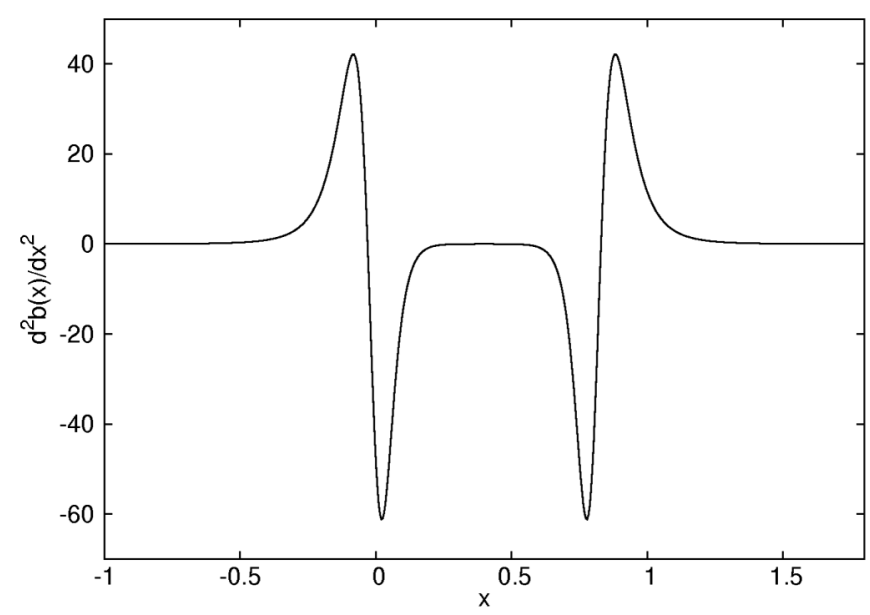

FIG. 5. Second derivative of the field profile for a dipole magnet with $a=0.1, b=0.4, d / a=0.5$, and $B_{0}=1$. 


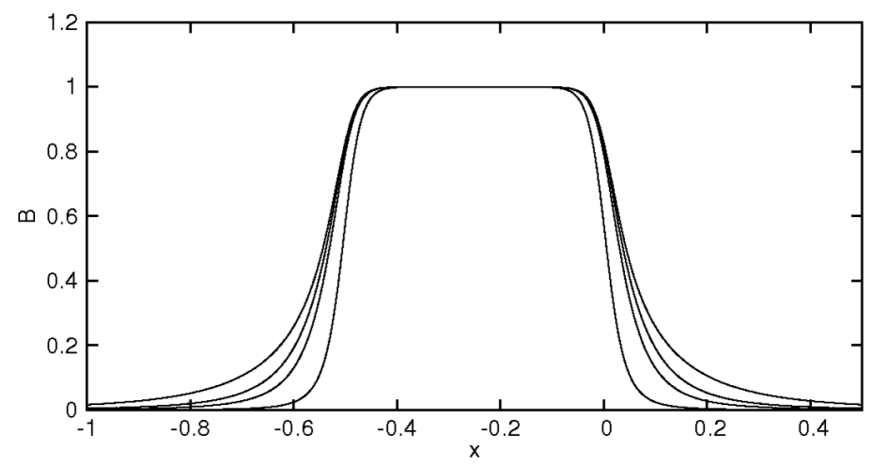

FIG. 6. Field profiles for dipole magnets with various values of $d / a$. In order from inner to outer curves, the $d / a$ values are 0.0 , 1.0, 2.0, and 5.0, respectively. All magnets have $2 a=0.1,2 b=$ 0.5 , and $B_{0}=1$.

for the same magnet. Figure 6 shows the field profiles for various values of $d / a$.

\section{COMPARISON WITH OTHER APPROACHES}

Other approaches to modeling soft-edge 2D dipoles have been already mentioned. In general, an analytic (in the complex-variables sense) function for the midplane field profile is required for generation of high-order maps. A possible problem with using some ad hoc analytic forms for the midplane field (e.g. the Enge function) is that the procedure can be mathematically unstable since one does not know a priori what effective sources in the complex plane produce the assumed midplane field. This can be problematic if the analytic form chosen turns out to have singularities in the complex plane that are too close to the midplane. This has not historically been a problem with the Enge function when the right coefficients are used, but in principle it could be problematic with a poorly chosen set of Enge coefficients, for which effective sources turn out to be inside of or close to the beam volume. Moreover, as previously mentioned, the Enge function does not have a realistic falloff of the fringe field at large distances from the pole faces. It will be shown in the following section that certain third-order geometric aberrations are sensitive to the rate of falloff of the fringe field. Going the other way, i.e., computing the midplane field and its derivatives from known sources outside of the beam volume, guarantees convergence of the field expansion to arbitrary order in the complex plane out to the known sources. This is automatically true with the conformal-map approach described above. It is also true when measured field data are represented by sources outside of the beam volume. A detailed discussion of the Helmholtz-theorem treatment of field data is beyond the scope of this paper, but can be found in Ref. [10]. The Helmholtz theorem [10] can also be used to get physical insight into the small differences between field profiles based on the conformal maps for the doubleand single-sided pole pieces. According to the Helmholtz theorem, the field outside of the pole faces can be reproduced by integrating open-boundary integral kernels over line-charge-density distributions on the pole boundaries (double layers are not needed since field lines are perpendicular to this particular surface). The charge density is just $\mathbf{B} \cdot \mathbf{N}$, where $\mathbf{N}$ is the outward normal to the pole-piece surfaces. In the case of the double-sided pole pieces of Eq. (12), the effective sources are all on the poles and the field expansion is good out to $y=a$ inside of the pole pieces and to much larger $y$ values for $x<-2 b$ or $x>0$. In the case of the profile of Eq. (17), which is derived from the single-sided map by reflection and translation, there are some sources on the line $y=a$ outside of the pole faces, but their strength falls off exponentially in $x$ distance from the pole faces with constant $y$. All this means in practice is that the field expansion cannot be pushed past $y=a$ for a small region of $x$ values just outside of the pole faces, so the region of convergence is almost as large as with the true double-sided map. It perhaps should be emphasized that, though it is straightforward to find the equivalent sources of the present conformal-map model, they never appear explicitly in any computer algorithms of the field model.

The analytic expression for the field derived above has a conformal map built into it, and the conformal map must be numerically inverted to compute the field and its derivatives. This does make the algorithm a little slower than it would be with use of an explicit analytic form, but still much faster than field routines based on numerically tabulated sources or fields on boundaries, over which one must sum or integrate integral kernels weighted by sources, or perform numerical Fourier transforms on the fly, etc. The use of an implicit analytic form is apparently the price that must be paid to get an analytic field profile that combines an exponential approach to the field between the poles with an inverse cubic field falloff at large distances from the magnet.

\section{IMPLEMENTATION OF THE MODEL IN MARYLIE/IMPACT}

The new soft-edge dipole model has an element-type code "cmdipole" in MARYLIE/IMPACT and has one more parameter $(d / a)$ than the tanh model. In addition to $d / a$, the user specifies the pole length $2 b$, entrance angle $\theta_{1}$, exit angle $\theta_{2}$, and full gap $2 a$. For any 2D dipole field profile, the entrance and exit angles are related to the field integral by the equation

$$
\sin \theta_{1}+\sin \theta_{2}=\frac{1}{B \rho} \int_{-\infty}^{+\infty} B_{y}(x, 0) d x,
$$

where $B \rho$ is the rigidity. The central field $B_{0}$ can then be derived from Eqs. (22) and (25).

As the default option, the element has built-in negative drifts and rotations to make the linear transfer matrix for the new soft-edge dipole element resemble as much as possible that of a hard-edge parallel-pole-face dipole 
with length $2 b$; i.e., the effect of the long integration lengths before and after the magnet is removed and the final coordinates of the transfer map are in a reference frame with the $z$ coordinate axis parallel to the output reference trajectory (this is also the default option for the tanh dipole element).

For comparison of dipole models, MLI was used to generate maps for a four-dipole chicane. Maps were generated using the new conformal-map dipole model, the double tanh dipole model [Eq. (5)], and the standard MLI hard-edge dipole element gbend. An Enge-function model was not available in MLI for comparison, but in view of the exponential (or faster) falloff of the fringe field of the Enge function, the tanh model should give 3rd-order maps similar to those of the Enge model when the dominant effective sources of the Enge function are at approximately the same distance $(a)$ from the midplane. The dipoles were given a pole length $2 b=10 \mathrm{~m}$ and a bending angle of $3.5^{\circ}$. The three drifts between the dipoles had a length of $10 \mathrm{~m}$. The particles were $1 \mathrm{GeV}$ electrons. In both cases a full dipole gap $2 a=0.025 \mathrm{~m}$ was used. For the cmdipole model, the parameter $d / a$ was set to 0.5 . Second-order Taylor-map coefficients for the two models, and also for the equivalent standard hard-edge MLI general bend element, differed only on the level of a few percent or less. In the case of third-order coefficients, comparison of the two soft-edge models with the standard hard-edge MLI model is inappropriate since 3rd-order geometrical terms are not treated in a completely consistent fashion in the MLI hard-edge dipole elements. In the comparison of the cmdipole and tanh dipole models, there were significant differences in the values of some third-order geometrical aberration coefficients, in particular in the seven $y$-plane coefficients $\left(y \mid y^{3}\right),\left(y \mid y^{2} P_{y}\right),\left(y \mid y P_{y}^{2}\right),\left(P_{y} \mid y^{3}\right),\left(P_{y} \mid y^{2} P_{y}\right),\left(P_{y} \mid y P_{y}^{2}\right)$, and $\left(P_{y} \mid P_{y}^{3}\right)$. In fact, in the maps for this particular test chicane example, the $y$-plane 3rd-order Taylor coefficients from the tanh model had the same signs as the corresponding ones from the cmdipole model but were all larger in magnitude by the same factor (a factor of approximately 1.488). An explanation of this systematic effect is a subject for future work.

\section{ACKNOWLEDGMENTS}

The author thanks Paul Channell for running MAXIMA to provide explicit expressions for the derivatives of Eq. (24) and Robert Ryne for modifying the code MARYLIE/IMPACT to incorporate the present dipole model.

[1] A.J. Dragt et al., MARYLIE 3.0 Users Manual, A Program for Charged Particle Beam Transport Based on Lie Algebraic Methods, 2000, available from the Physics Department, University of Maryland, College Park, Maryland 20742-4111.

[2] R. Ryne et al., MaryLie/IMPACT Manual: A Parallel Beam Dynamics Code with Space Charge based on the MaryLie Lie Algebraic Beam Transport Code and the IMPACT Parallel PIC Code, available from, Lawrence Berkeley National Laboratory, CA.

[3] M. Berz, J. Hoefkens, and K. Makino, Technical Report No. MSUHEP-20703, 2002; see also http://cosy.pa .msu.edu.

[4] A. J. Dragt, D. R. Douglas, L. M. Healy, F. Neri, G. Rangaranjan, and R. Ryne, Annu. Rev. Nucl. Part. Sci. 38, 455 (1988).

[5] H. Enge, Focusing of Charged Particles, edited by A. Septier (Academic Press, New York, 1967), Vol. II, pp. 240-241.

[6] N. Davy, Philos. Mag. 35, 819 (1944).

[7] N. Barton, J. Aust. Math. Soc. Series B, Appl. Math. 26, 404 (1985).

[8] L. M. Milne-Thomson, Elliptic Integrals, Handbook of Mathematical Functions, edited by M. Abramowitz and I. A. Stegun (Dover Publications, New York, 1972, Chap. 17.

[9] W.H. Press, B. P. Flannery, S. A. Teukolsky, and W. T. Vetterling, Numerical Recipes (Cambridge University Press, New York, 1989).

[10] A. J. Dragt, P. Roberts, T. J. Stasevich, A. Bodoh-Creed, and P. L. Walstrom, arXiv:1012.1647v1. 